\title{
ASME Code Data Report for the CC Cryostat
}

\author{
Engineering Note \# 3740.214-EN-265
}

Issued: November 6, 1990

Originator: Rick Luther 
November 11, 1987

Mr. Joe R. Sloan

CBI Na-Con, Incorporated

24137 111th Street

Plainfield, Illinois 60544

\section{SUBJECT: ASME Code Documentation \\ CC Cryostat Head Removal and Replacement \\ CBI Na-Con Contract C70708 \\ Fermilab Contract 938570}

Dear Joe:

Attached per your request are the ASME Code Data Reports for the CC Cryostat. Included are:

- Form U-1 for the inner vessel of the Cryostat.

- Rubbings of the Code nameplate and the duplicate nameplate located on the top of the outer vessel.

- Forms U-2 for the heads for both vessels (inner and outer).

If you need any additional documentation please let me know.

Very truly yours,

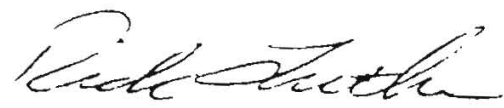

Richard D. Luther

$D \emptyset$ Cryogenics Engineering

Mail Station 357

(312) 840-2322

$\mathrm{RDL} / \mathrm{hs}$

cc: Mulholland

Luther/File: CC Head Removal/Replacement 
1. Manutaciured and cerrified by Richmond-Lox Equipment Company, Hwy 25 South, Delphi, IN 46923 2. Manutactured tor Fermi National Accelerator Lab, P.0. Box 500, Batavia. IL 60510

3. Location of installation Same

4. Type Horiz. Jackt. Torus 30093

(Name and address)

5. The chemical and physical properties of all parts meet the requirements of material specifications of the ASME Boiler and Pressure Vessel Code The design, construction, and workmanship conform to ASME Rules, Section VIII. Division 1 Winter, 1986

Winter 1986

Addends idsel - Cose Case No.

llems 6-1" incl. to be completed for single wall vessels, fackets of jacketed vessels. or sheets of heat exchangers

6. Shell: Non-Code

Matl. ISpec No. Gradet Tom Tha tin) - Corr Allow. lin.1

7. Seams

8. Heads: (a) Mart

\begin{tabular}{|c|c|c|c|c|}
\hline & $\begin{array}{l}\text { Location iToo. } \\
\text { Bottom. Ends) }\end{array}$ & $\begin{array}{l}\text { Minimum } \\
\text { Thickness }\end{array}$ & $\begin{array}{l}\text { Corrosion } \\
\text { Allowance }\end{array}$ & $\begin{array}{l}\text { Crown } \\
\text { Radius }\end{array}$ \\
\hline (a) & & & & \\
\hline (b) & & & & \\
\hline
\end{tabular}

14 removable, bolts used (describe other fastenings)

9. Type of Jacket

10. Jacket Closure

11. MAWP Describe as ogee 8 weid. bar. elc. (b) Matl.

Fi (Spol, Purtedil, or full)

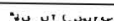


FORM U.4 MANUFACTURER'S DATA REPORT SUPPLEMENTARY SHEET As Required by the Provisions of the ASME Code Rules. Section VIII. Division 1

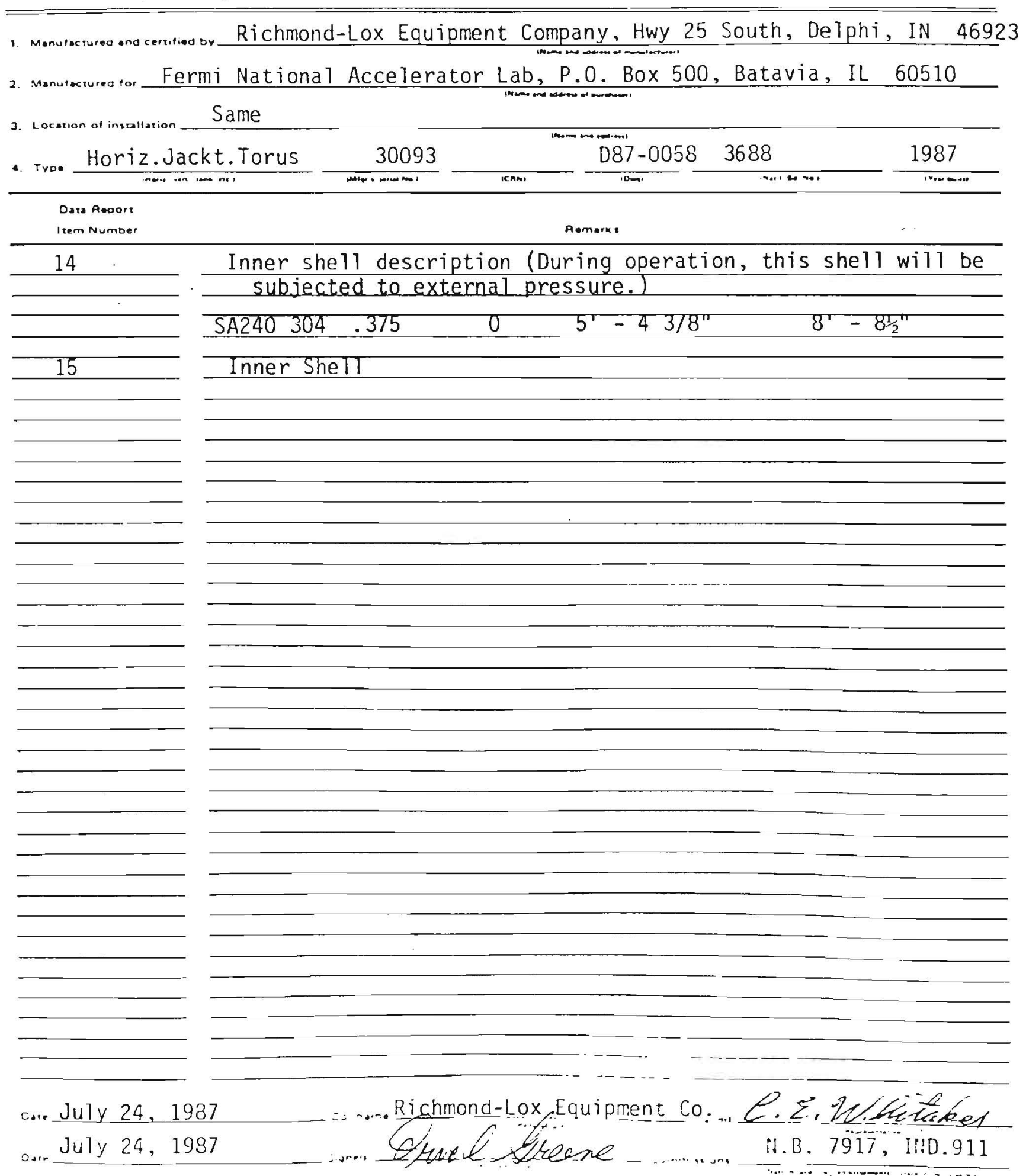




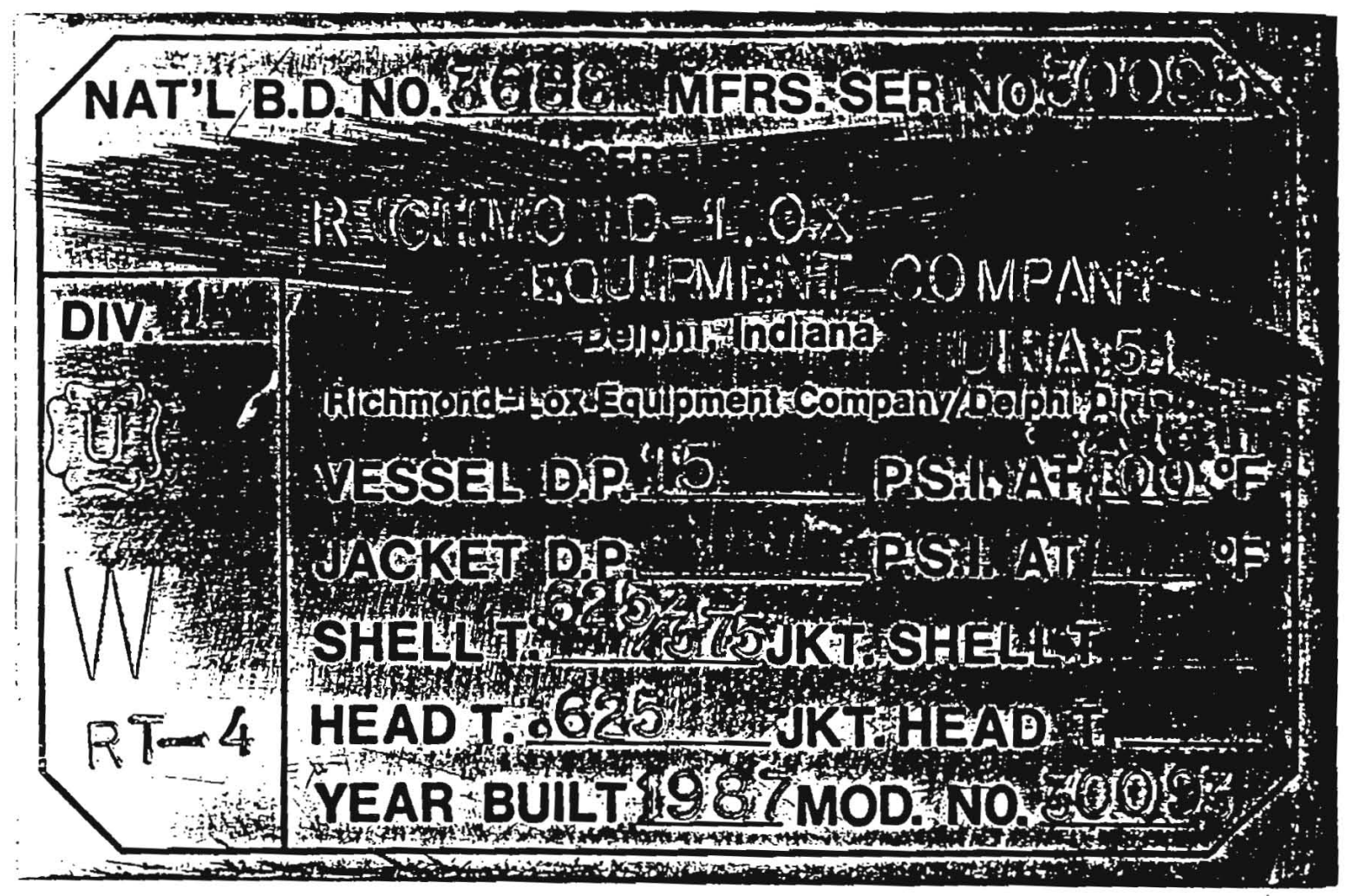


ond

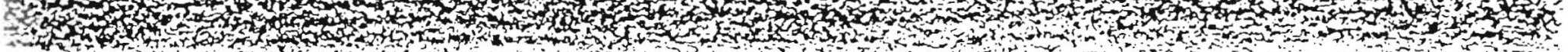

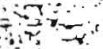
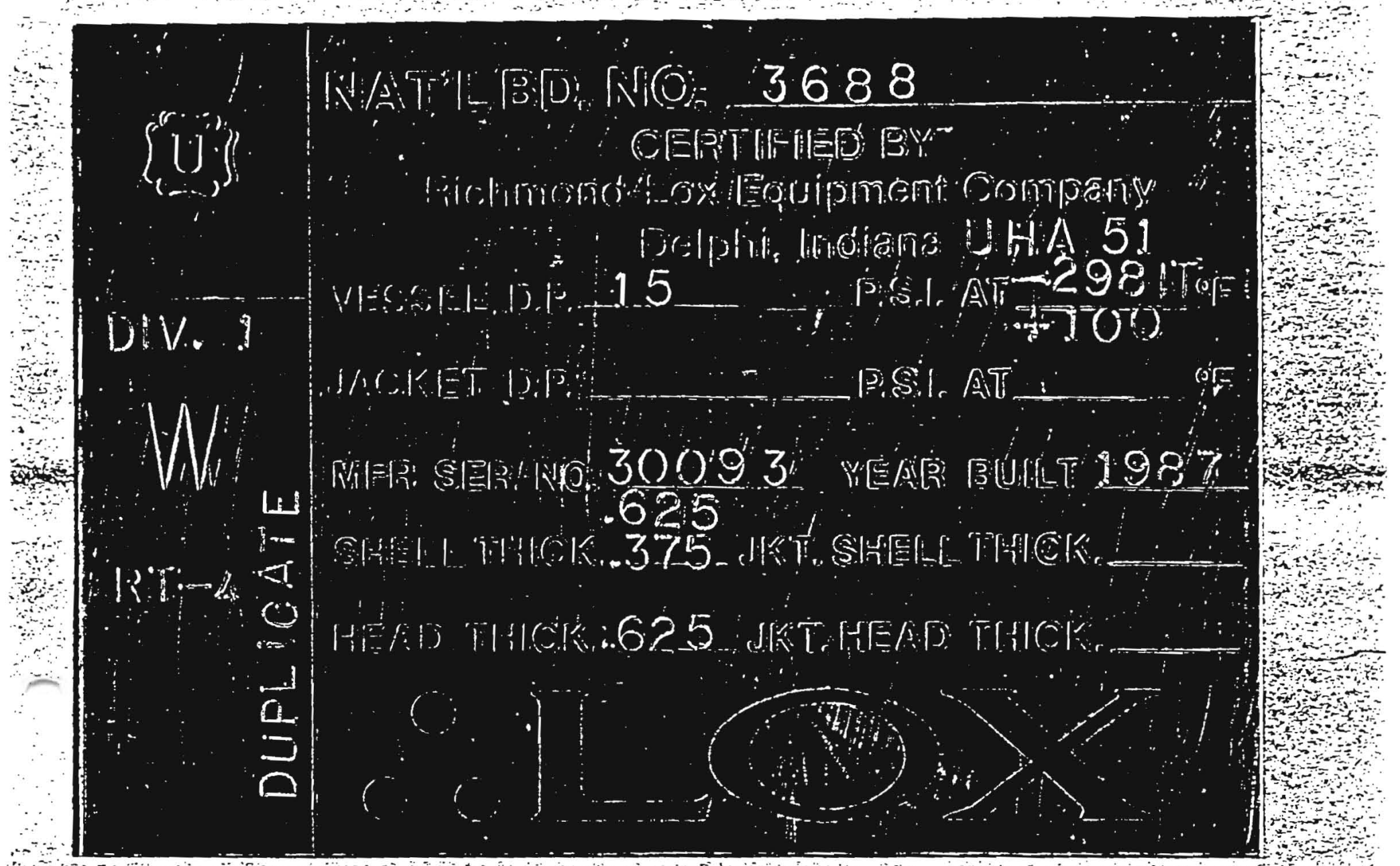

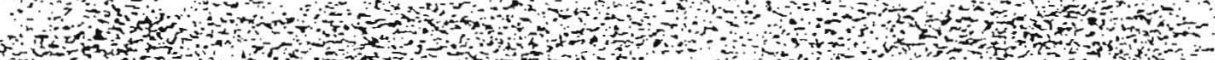

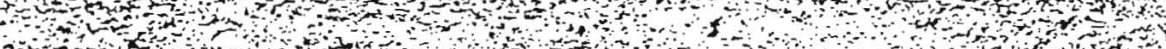

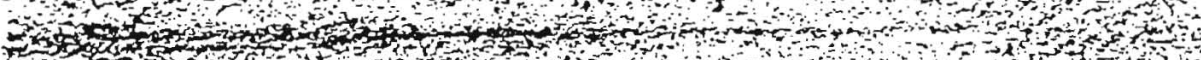

T.

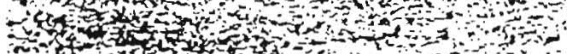

$\rightarrow x^{2}$

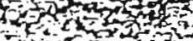

Now

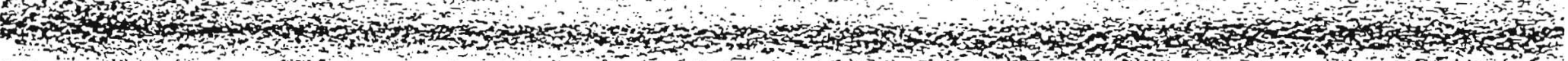

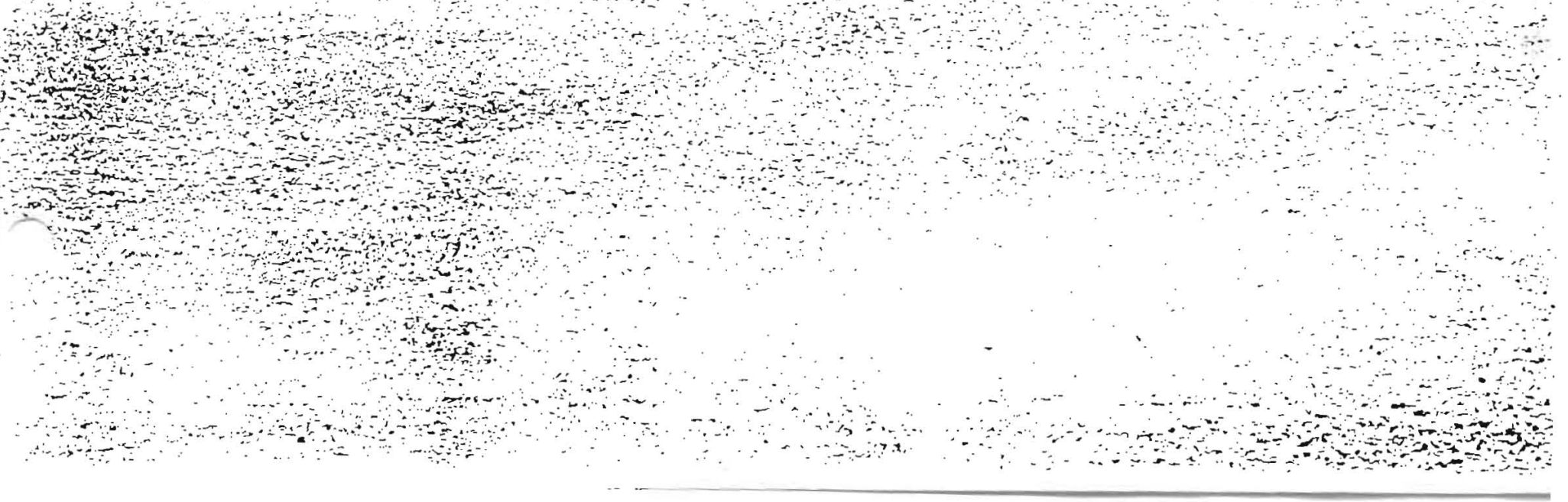


FORM U-2 MANUFACTURER'S PARTIAL DATA REPORT

A Part of a Pressure Vessel Fabricated by One Manufacturer for Another Manufacturer

As Required by the Provisions of the ASME Code Rules, Section VIII, Division 1 1. Manutactured and certitied by The Stacey Mfg. Co., 259 Township Ave., Cincinnati, Ohio 45216

. Manufactured for Richmond-Lox Equipment Co., Delphi, Indiana 46923

3. Location of installation

Not known

4. Type-Horizontal Tank

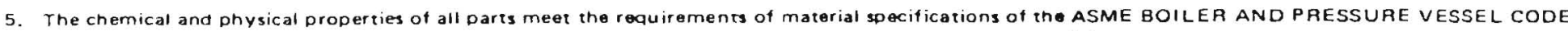
The design, construction, and workmanship conform to ASME Rules, Section VIII, Division 111986

December 31, 1986

$$
\text { Addends } 10 \text { actel }
$$

6. (a) Drawing propared by The Stacey Mfg. Co.

(b) Description of part inspected 16 $1-1 / 2^{\prime \prime}$ OD Half Torust

7. Postweld heat treatment: Tomp.

${ }^{\circ} \mathrm{F}$ Time

ltems 8-13 incl. to be completed for single wall vessels, jackets of jacketed vessels, or shells of heat exchangers

8. Shell:

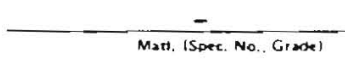

9. Seams:

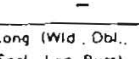
Singl.. Lap, Bute)

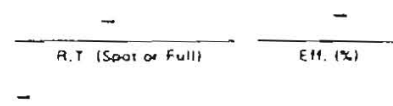

$-$

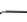

ispere. No., Gisoel

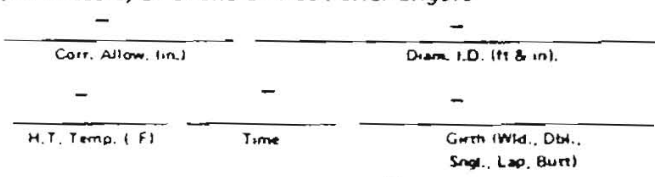

(b) Matl.

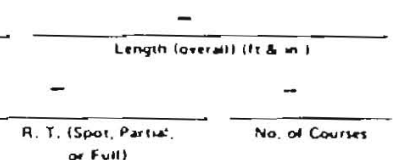

10. Heads: (a) Matl.

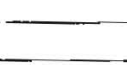

\begin{tabular}{|c|c|c|c|c}
\hline $\begin{array}{l}\text { Location (Top. } \\
\text { Eotrom. Ends) }\end{array}$ & $\begin{array}{l}\text { Minumum } \\
\text { Thickness }\end{array}$ & $\begin{array}{l}\text { Corioswan } \\
\text { Allowance }\end{array}$ & $\begin{array}{l}\text { Crown } \\
\text { A2dius }\end{array}$ \\
\hline (a) & - & - & - & - \\
\hline (b) & - & - & - & - \\
\hline
\end{tabular}

If removable, bolts used (describe other fastenings)

\begin{tabular}{|c|c|}
\hline US & $\begin{array}{l}\text { Knuckle } \\
\text { Radius }\end{array}$ \\
\hline & - \\
\hline & - \\
\hline
\end{tabular}

\begin{tabular}{c|c|}
$\begin{array}{c}\text { Elliotical } \\
\text { Ratio }\end{array}$ & $\begin{array}{c}\text { Conical } \\
\text { Adex Angie }\end{array}$ \\
\hline- & - \\
\hline- & - \\
\hline
\end{tabular}

\begin{tabular}{|c|c|}
\hline \multicolumn{2}{|c|}{ ISpec No., Grade) } \\
\hline $\begin{array}{l}\text { Hemisphericas } \\
\text { Aadius }\end{array}$ & $\begin{array}{c}\text { Flat } \\
\text { Diameter }\end{array}$ \\
\hline - & - \\
\hline - & - \\
\hline
\end{tabular}

Fuil!

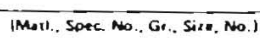

11. Type of Jacket

12. Jacket Closure. If botted, describe or sketch.

13. MAWP -

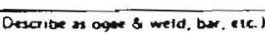
Proof Test $-$ Hydro., pneu., or comb. test press. psi at max. temp. ${ }^{\circ} \mathrm{F}$. Min. temp. (when less than $-20^{\circ} \mathrm{F}$ ). psi. fems 14 and 15 to be completed for tube sections

14. Tubesheets:

$$
\frac{-}{\text { Scatronsey Mall. ISpect No, Gr.) }}
$$
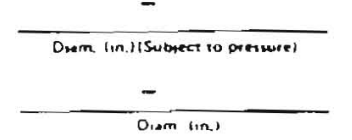
If bar, give dimensions

$-$

$$
\text { Mont. (Spare. No.. Grose) }
$$
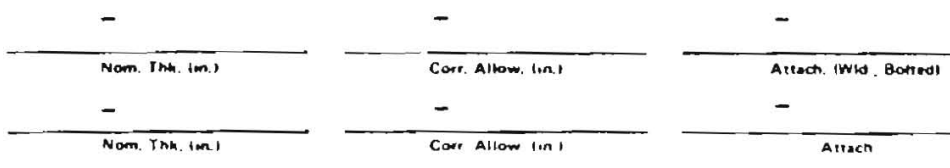

15. Tubes:
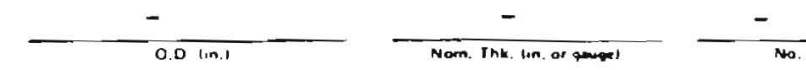

Irems 16-18 incl. to be.completed for inner chambers of jacketed vessels or channels of heat exchangers

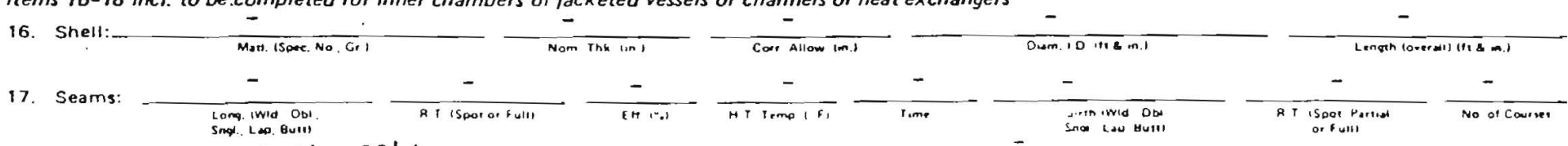

18. Heads: (a) Matt..SA240-304: (b) Marl.

iswer No. Lirsol

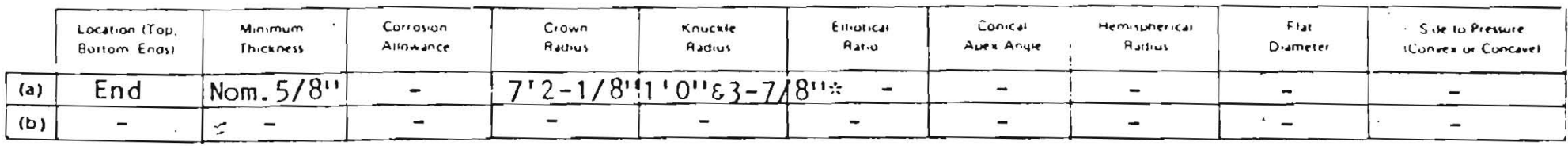

if removable, bolts used (describe other tastenings) 
7. MAWP Dsi at max. temp.

- F. Min temp. (when less than $-20^{\circ} \mathrm{F}$ ) $\stackrel{\circ}{F}$.

Hydro., pneu. or comb. test press psi.

Items below to be completed for all vessels where applicable

20. Nozzles, Inspection and Safery Valve Openings:

\begin{tabular}{|c|c|c|c|c|c|c|c|c|}
\hline 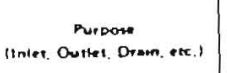 & No. & Oorm. & Trose & Most. & $\begin{array}{l}\text { Nom. } \\
\text { Thin. }\end{array}$ & $\begin{array}{l}\text { Aemioucement } \\
\text { satl. }\end{array}$ & $\begin{array}{l}\text { How } \\
\text { Altixsed }\end{array}$ & Locution \\
\hline- & $\overrightarrow{-}$ & - & - & - & - & - & - & - \\
\hline- & - & - & - & - & - & - & - & - \\
\hline- & - & - & - & - & - & - & - & - \\
\hline- & - & - & - & - & - & - & - & - \\
\hline- & - & - & - & - & - & - & - & - \\
\hline- & - & - & - & - & - & - & $=$ & - \\
\hline- & - & - & - & - & - & - & - & - \\
\hline- & - & - & - & - & - & - & - & - \\
\hline- & - & - & - & - & - & - & - & - \\
\hline- & - & - & - & - & - & - & - & - \\
\hline- & $=$ & - & - & - & - & - & - & - \\
\hline- & - & - & - & - & - & - & $=$ & $=$ \\
\hline- & - & $=$ & - & $\simeq$ & - & - & - & - \\
\hline- & 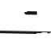 & - & - & $=$ & - & - & - & - \\
\hline- & - & - & $=$ & - & - & - & - & - \\
\hline- & - & - & - & - & - & - & - & - \\
\hline
\end{tabular}

21. Supports: Skirt

(Yrom Lugs $\frac{-}{\text { (No.) }}$ (No.) IDencribet Arrached

iwnere and now

Remarks: Neither design nor hydrostatic testing were performed by The Stacey Mfg. Co. *This half section of $16^{\prime} 1-1 / 2^{\prime \prime}$ OD $\times 5^{\prime} 11-3 / 4^{\prime \prime}$ 10 Torus is a ten-piece construction and consists of one (1) latitudinal double butt-welded joint at reverse $0^{\prime} 3-7 / 8^{\prime \prime} / \mathrm{KR}$ tangent point jointing $7^{\prime} 2-1 / 8^{\prime \prime}$ IR donut portion, four (4) meridinal double butt-welded joints at $5^{\prime} 11-3 / 4^{\prime \prime} 10$ reverse $0^{\prime} 3-7 / 8^{\prime \prime}$ inside knuckle portion $\&$ twelve (12) meridinal double butt-welded joints at $7^{\prime} 2-1 / 8^{\prime \prime}$ IR donut with $1^{\prime} 0^{\prime \prime} I K R$ portion. All welding Ful R.T.

\section{CERTIFICATE OF SHOP COMPLIANCE}

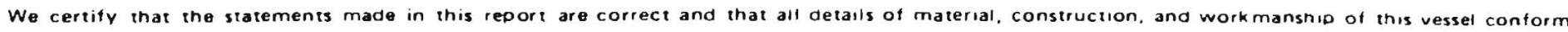
to the ASME Code for Pressure Vessels. Section VIII. Division 1.

"u" Certificate of Authorization No. 1830

Date 3 , 8 ?

co. name The Stacey Mfg. Co.

$$
\text { expires }
$$

CERTIFICATE OF SHOP INSPECTION

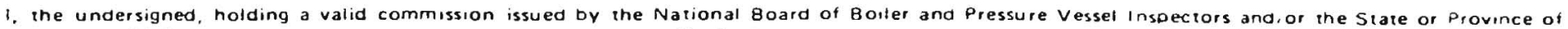
Ohio

of Hartford, Conn. have inspected the pressure vessel described in ihis Manufacturer's Partial Data Report on _. . 3 . $/$

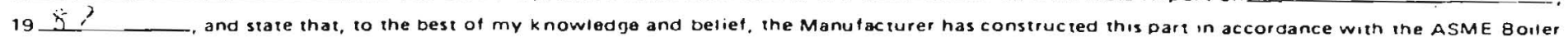

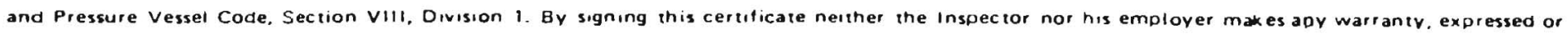

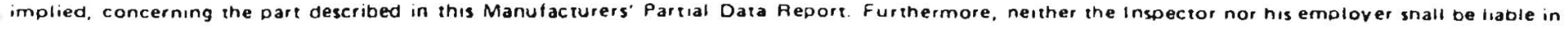
any manner for anv bersonal injury or property damage og a loss of any $k$ ind aysyg trom or connected with this inspection.

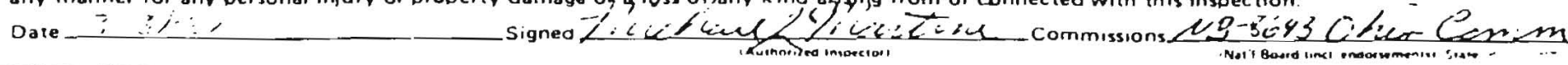


FORM U-2 MANUFACTURER'S PARTIAL DATA REPORT

A Part of a Pressure Vessel Fabricated by One Manufacturer for Another Manufacturer

As Required by the Provisions of the ASME Code Rules, Section VIII, Division 1

Manutactured and certified by

The Stacey Mfg. Co., 259 Township Ave., Cincinnati, Ohio 45216 (N ame and adders or monutectureel

2. Manutactured for

Richmond-Lox Equipment Co., Delphi, Indiana 46923

3. Location of instaltation

Not known

4. Type Horizontal Tank 6309B

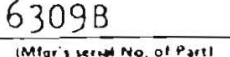

$0-44-A 53$ Rev. 2

ICANI

$\frac{0-44-A 53 \text { Rev. } 2}{10 \text { ram Nol }}$

INAT' Bo NOP

1987

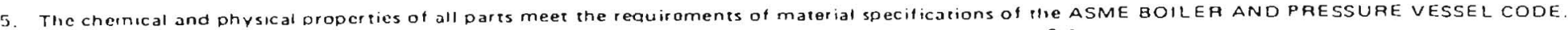
The design, construction, and workmanship conform to ASME Rules, Section VIII, Division 1 _ 1986

December 31,1986 Addends (OAate) Code Case No. Specist seence per UC 120101

6. (a) Drawing prepared by The Stacey Mfg. Co. (b) Description of part inspected

7. Postweld heat treatment: Temp. ${ }^{\circ} \mathrm{F}$ Time _ -

Itenis 8-13 incl. to be completed for single wall vessels, jackets of jacketed vessels, or shells of heat exchangers

8. Shell: Mati. ISpec. No., Grodel

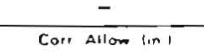
D.sin 1.D. (n) sin).

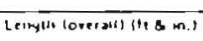

9. Searns: Long. IWid. Obt.,
Sngl., Lap. Butil $\frac{-}{\text { A.T. } 15 \text { prot or full }}$ $\frac{-}{\text { EHI. } 1 \% 1} \frac{-}{\text { HT Temp. (F) }}$ $\frac{-}{\text { Time }}$ Gurih isida. Dtt.
Sirgl. Lad. Buris (b) Mail. $-$

10. Heads: (a) Matl. H. T. ISvot. Poit
on Full] $\frac{-}{\text { No. of Couses }}$

\begin{tabular}{|c|c|c|c|c|}
\hline $\begin{array}{l}\text { Locstion (Tuis. } \\
\text { Buttorn. E nebs) }\end{array}$ & $\begin{array}{l}\text { Misumuin } \\
\text { Thiekterss }\end{array}$ & $\begin{array}{l}\text { Curressun } \\
\text { Allowance }\end{array}$ & $\begin{array}{l}\text { Corinus } \\
\text { Ractius }\end{array}$ & $\begin{array}{l}\text { Kourkir } \\
\text { Adtius }\end{array}$ \\
\hline - & - & - & - & - \\
\hline - & - & - & - & - \\
\hline
\end{tabular}

If removable, bolts used (describe other fastenings) $-$

\begin{tabular}{|c|c|c|c|c|}
\hline $\begin{array}{l}\text { Elturtical } \\
\text { Aalio }\end{array}$ & 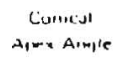 & 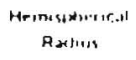 & $\begin{array}{c}\text { Fl.se } \\
\text { D.seneter, }\end{array}$ & $\begin{array}{l}\text { Sulke to Ptriwiter } \\
\text { ICusives in Concaves }\end{array}$ \\
\hline - & - & - & - & - \\
\hline- & - & - & - & - \\
\hline
\end{tabular}

AMitil, Surec. Nu GT., Sode. No.1

11. Type of Jacket ___ -

12. Jacket Closure _ _ -

If bolted, describe or sketch

toescribe as oave \& weld, bas. elc

Proof Test

$\cdot$

13. MAWP -

Dsi at max. temp.

"F. Min. temp. (when less than $-20 " \mathrm{~F}$ ) "F.

Hydro., pneu., or comb. test press.

$-$ usi

lems 14 and 15 to be completed for tube sections

14. Tubesheets:
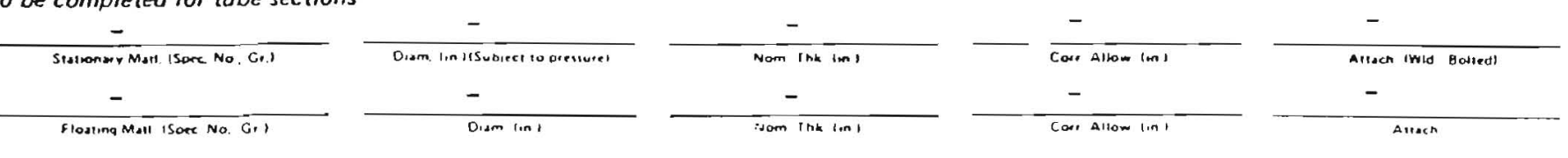

15. Tubes:

$$
\text { Matt. ISver. No., Grade) }
$$

$0.0 \ln 1$
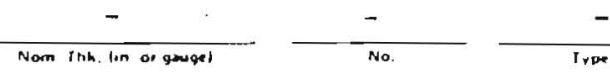

ltems 16-18 incl. to be completed for inner chambers of jacketed vessels or channels of heat exchangers 16. Shell: Msti (spec. No. $\mathrm{C}_{\text {I }}$ ) Nom Itix in.t
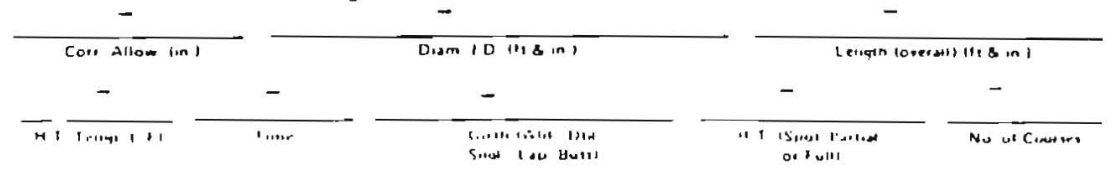

17. Seams

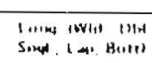

H T ispur un I vill

(b) Mar

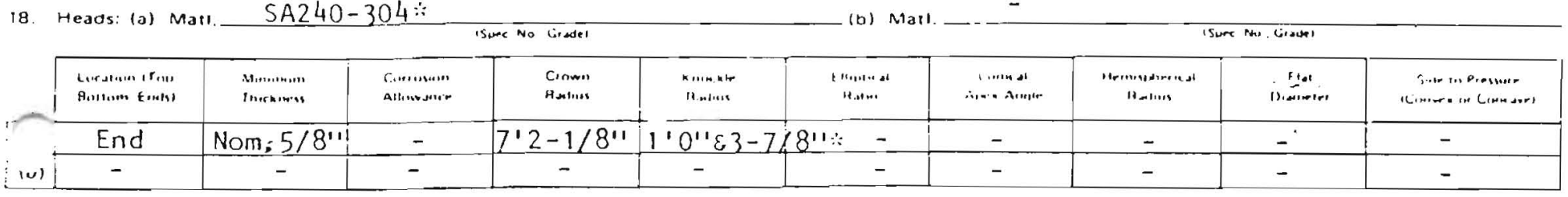

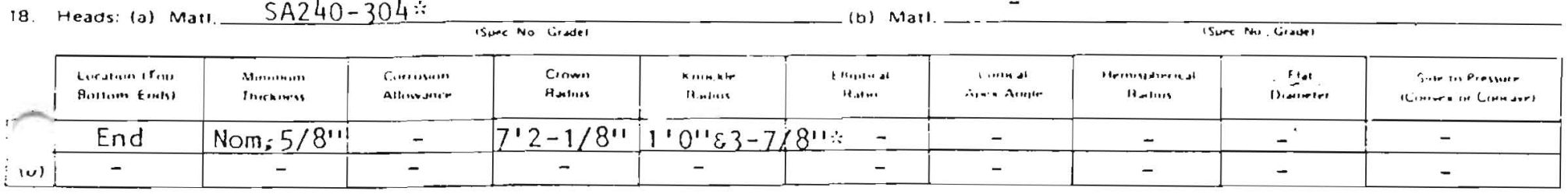

ISure Phe. Geaser)

If removable bolis used (describe niner tasennmal 
Division or Egtmat comporation

THINK FIRST ... THINK EASTERN

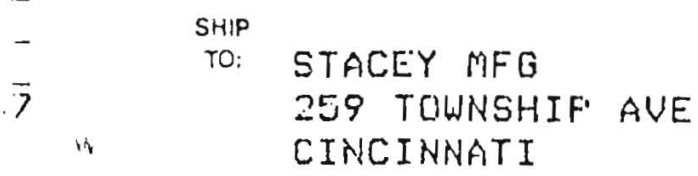

DIFIECL

WILLIAISS AND COMFANY INC. 901 FENMSYLUANIA AUENUE

OH 45216 PITTSBURGH
CERTIFIED MATERIAL TEST REPORT We cerity that oll of the test resulls and ine stalements of Deriarmed operations racorded aere are

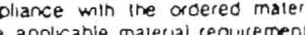

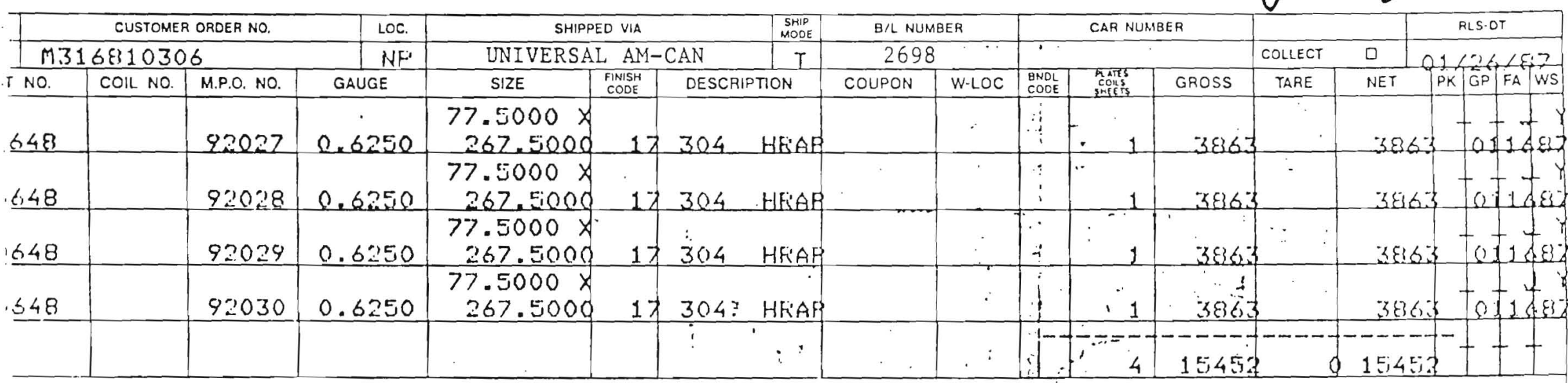

ECS ASTM A240-84A ASME SA240 SECT II SB5 ADD

$$
\begin{gathered}
016036-6309 \\
1 / 29 / 87 \\
\text { ots. }
\end{gathered}
$$$$
6309
$$$$
41-1
$$

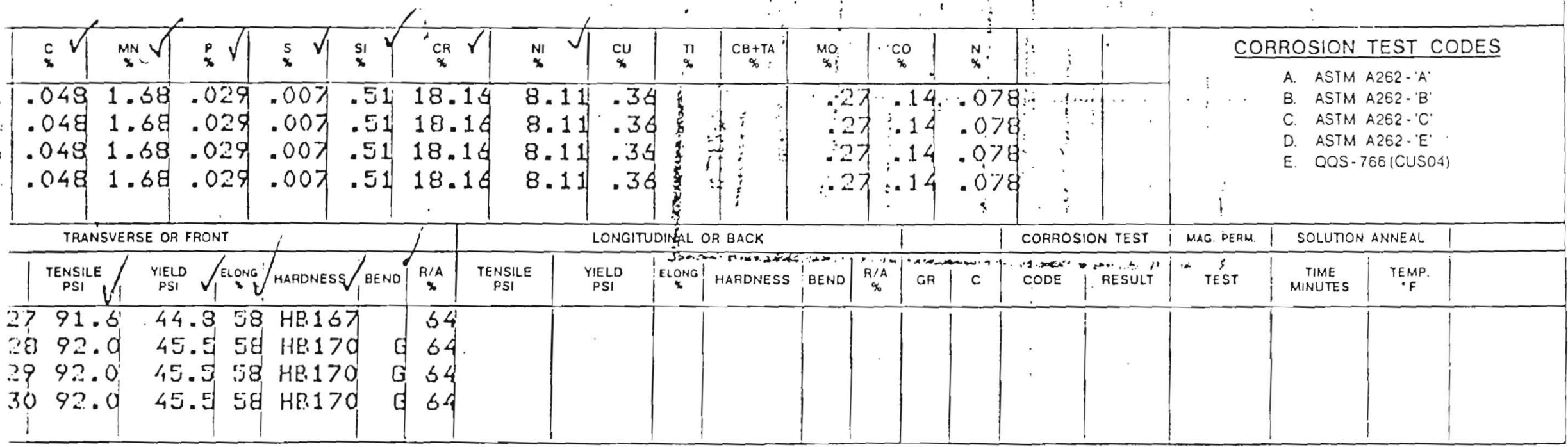




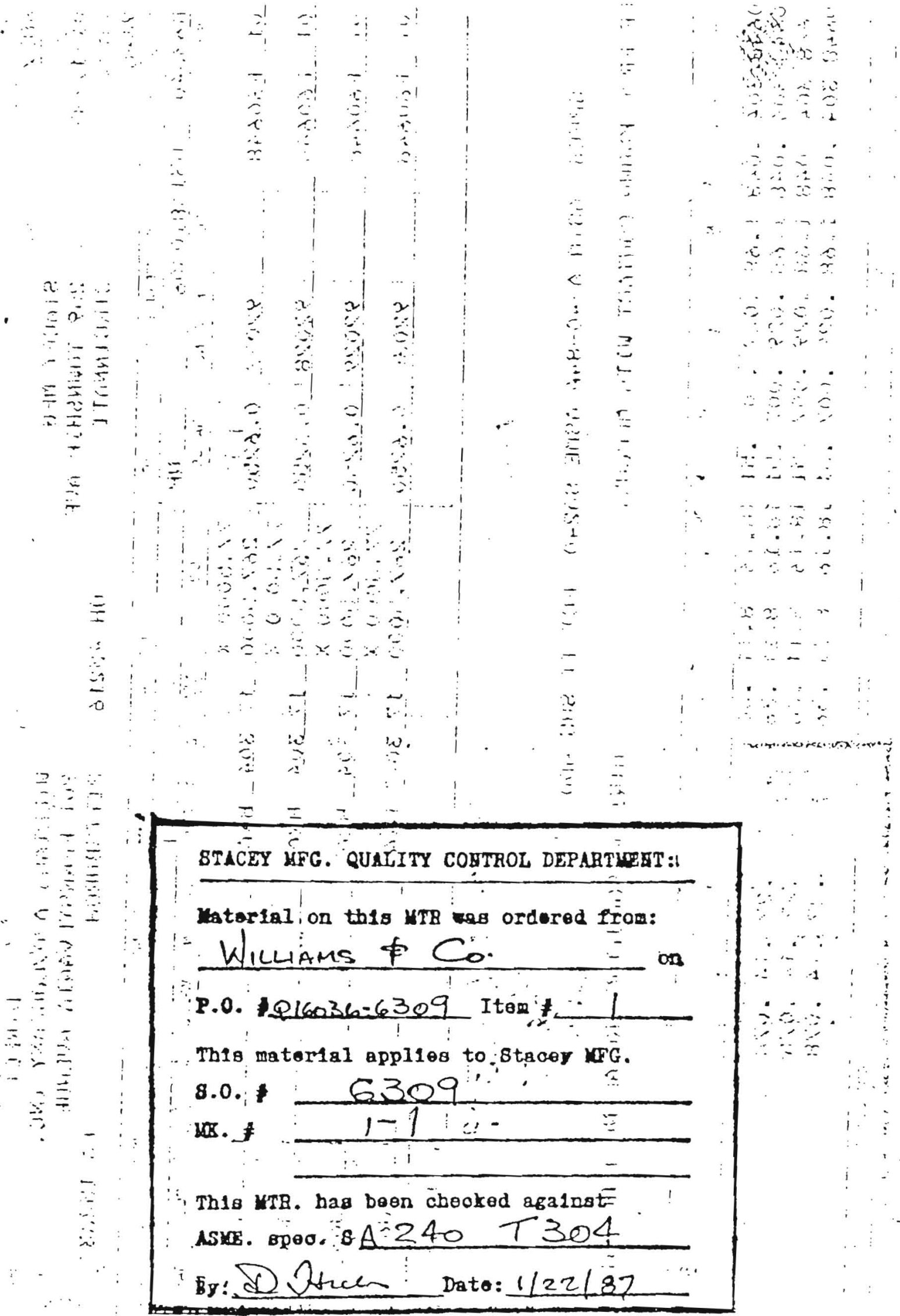


JESS

TASME

COMF

$Q Q-S-$

Heat

15647

1504 L

$1564 \varepsilon$

Heat

1564 :

$1564 \varepsilon^{\prime}$

Hiest

1564 :

-

$1564 i$

MAGTHF. MATEF

\section{STEEL COMPANY}

Washington, Pennsylvania 15301
OUR ORDER NO. YOUR ORDER NO. CERTIFICATE OF TEST MEMONO
आमUतय, CU

$71611-99-5): 06$ 42534

$10 / 06 / 86$

$06 / 86$

Stif to: WILLIAMS \& CO 3640 FEINHOLD MR INAINNATI OH

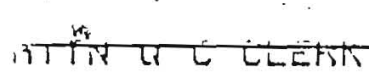

45237

FITTSEURIGH F'A

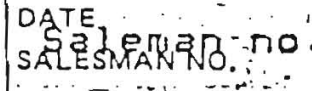

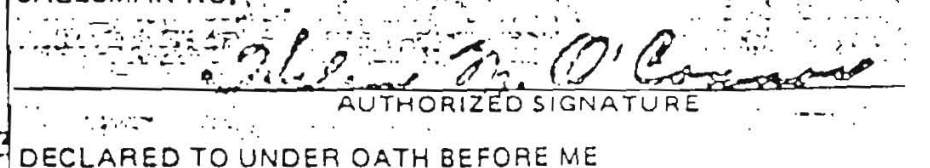

\section{304 STAINLESS HRAF}

-240-86 AMS 551311. ASTM A240-85a . ASTM A1S7-843

15233 DECLARED TO UNDER OATH BEFORE ME

ASTM A312-85 COMFOSITION \& MECHANICAL: FROFEFTIES TO ASTM A276-85a

SC AMENII 6 COND A
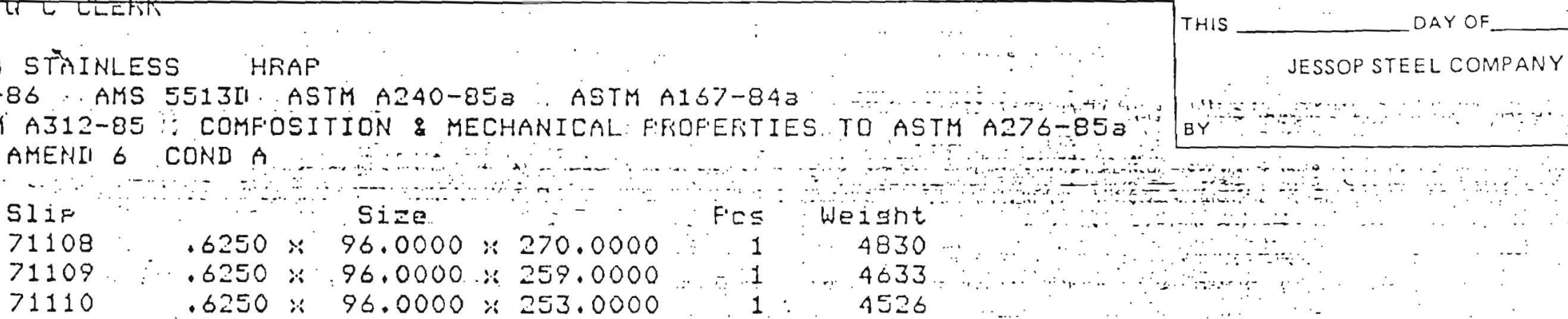

Slif

71108

71109

71110

\section{Size}

$.6250 \%$ $.6250 \cdots$

$96.0000 \because 270.0000$

F.CS

Weisht

4830

$.6250 \% 96.0000 \% 253.0000$

4633

4526

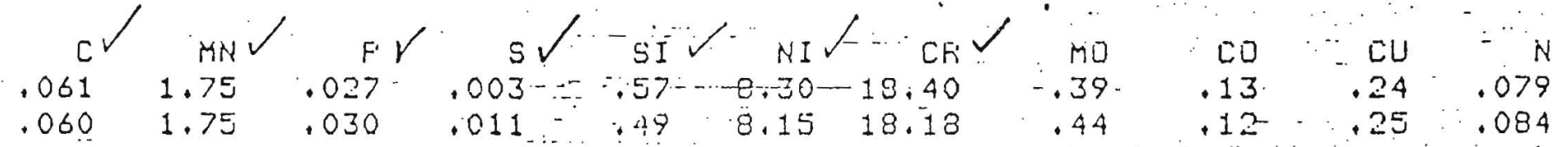

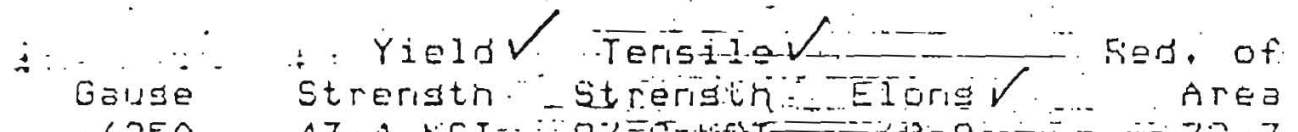

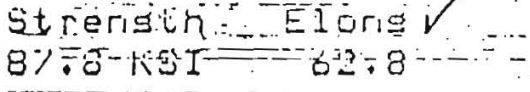

Hardriess

$43.4 \mathrm{KSI}$

LitN1 170

or

Corrosion

ロバ

$43.0 \mathrm{KISI}$

$3: 4 \times 5+2=0 \div 1$

71

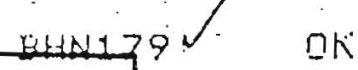

$\underline{n K}$

STACEY YEG. QUALITY CONTROL DEPARTKENT:

C FETIMEASILITY-LESS THAN .02

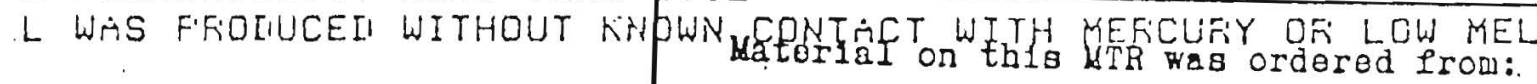

Wulars \& Co

on

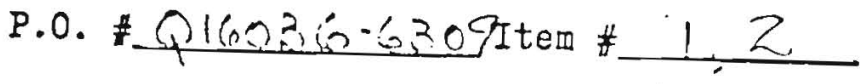

This material applies to Stacer WFG.

s.o. \# $\quad 6309$

1KK. \#

, $1-3$

Th1s HTR. has been checked against

ISUE.. speo. S A 240 T.301

(FOD

FOIHT CONTAMIHPMTS

Grai

$S i=$

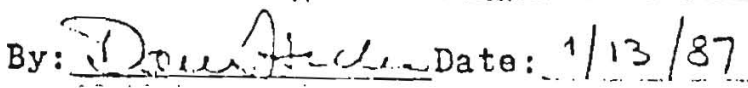




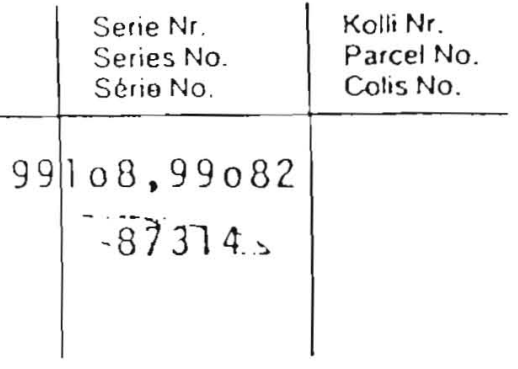

Chemische Analyse - Chemical Analysis - Analyse chimique

\begin{tabular}{|c|c|c|c|c|c|c|c|c|c|c|c|}
\hline $\begin{array}{l}\text { Charge } \\
\text { Heat } \\
\text { Coulee }\end{array}$ & $\begin{array}{l}\text { Serie } \\
\text { Serie } \\
\text { Serie }\end{array}$ & $\underset{\%}{\mathrm{c}} \sqrt{ }$ & $\underset{\%}{\mathrm{Si}_{\%}}$ & $\begin{array}{c}\mathrm{Mn} \\
\%\end{array}$ & $\begin{array}{l}\mathrm{p} \\
\%\end{array}$ & $\mathrm{~s}_{\%}^{\mathrm{s}} \sqrt{ }$ & $\begin{array}{l}\mathrm{Cr}_{\%} \\
\%\end{array}$ & $\begin{array}{lll}\text { Mo } & V \\
\% & V\end{array}$ & $\begin{array}{l}\mathrm{Ni} / \\
\because\end{array}$ & $\mathrm{Cu}^{\prime} \%$ & $\begin{array}{l}\text { Nb } \\
\%\end{array}$ \\
\hline & 108 & 0,016 & 0,65 & 0,78 & 0,024 & 0,014 & 20,06 & 0,33 & 10,20 & 0,090 & \\
\hline & 082 & 0,018 & 0,69 & 0,79 & 0.024 & 0,011 & 20,28 & 0,04 & 9 , & 0,060 & \\
\hline & 314 & 0,028 & 0,73 & 0,74 & 0,027 & 0,011 & $\mid 9.91$ & 0.26 & 10,2 & 0,18 & \\
\hline
\end{tabular}

Mechanische Gütewerte - Mechanical Properties - Propriétées mécaniques

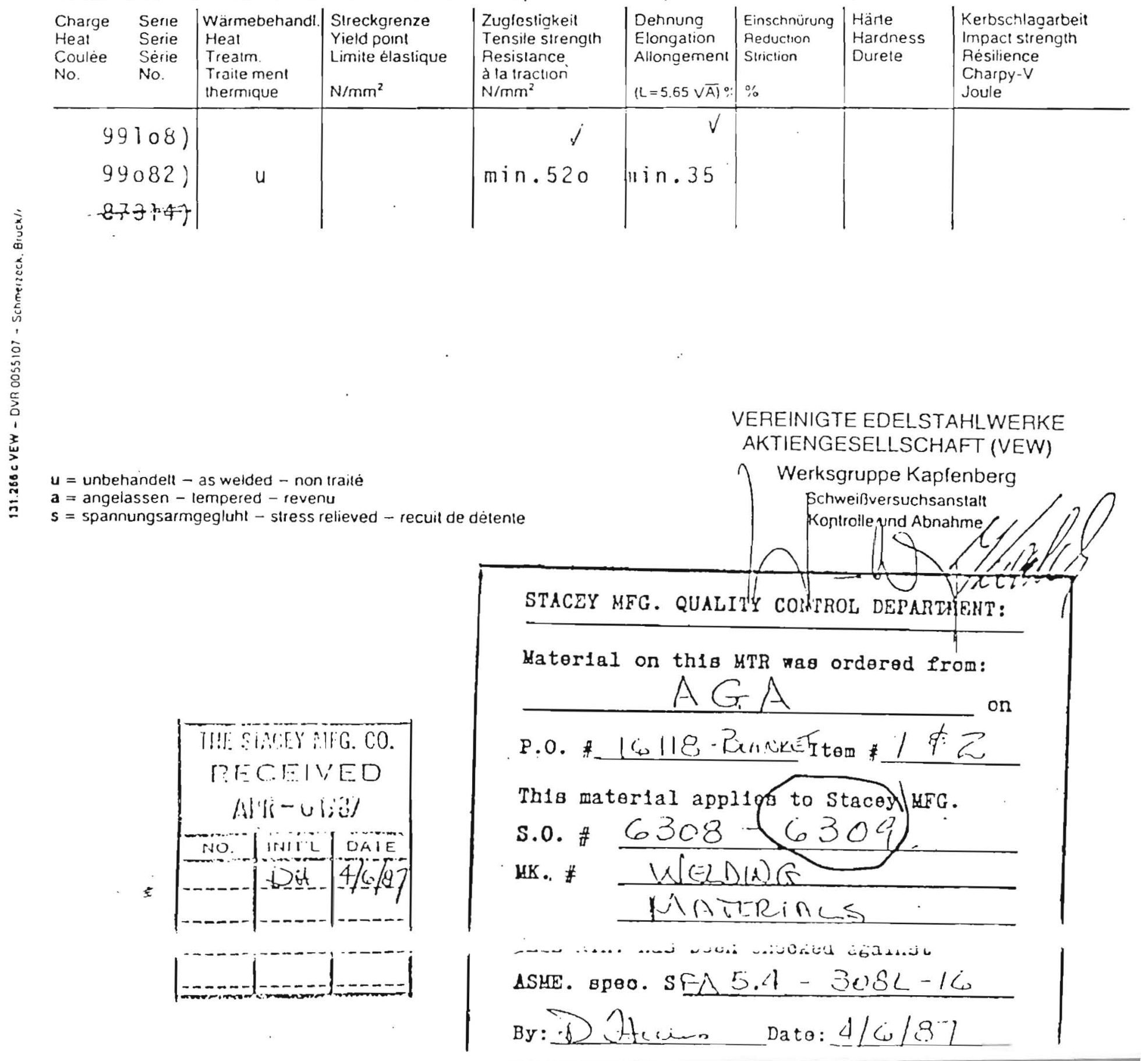


FORM U-2 MANUFACTURER'S PARTIAL DATA REPORT

A Part of a Pressure Vessel Fabricated by One Manufacturer for Another Manufacturer

As Required by the Provisions of the ASME Code Rules, Section VIII, Division 1

1. Manufactured and certitied by The Stacey Mfq. Co., 259 Township Ave., Cincinnati, Ohio 45216

2. Manufactured for

Richmond-Lox Equipment Co., Delphi, Indiana 46923

3. Location of installation

Not known

4. Type Horizontal Tank

6308A

|torix a vert rankt

(Mitgris seriat No. of Part)

(CAN)

(ad adrosi)

$0-43$ A53 R. 2

$\frac{-}{\text { (Nati. Bd. No.) }}$

1987

5. The chemical and physical properties of all parts meet the requirements of material specifications of the ASME BOILER AND PRESSURE VESSEL CODE. The design, construction, and workmanship conform to ASME Rules, Section VIII. Division 1_ 1986

December 31, 1986

Addendo (Oale) Cose case No

Specisl service per UG.1201d

(a) The Stacey Mfg. Co. (b) Description of part inspected $16^{\prime} 11-3 / 4^{\prime \prime}$ OD Half Torus:

7. Postweld heat treatment: Temp. ${ }^{\circ} \mathrm{F}$ Time _-

Items $8-13$ incl. to be completed for single wall vessels, jackets of jacketed vessels, or shells of heat exchangers

8. Shell:

Mati. (spec No., Grade)

Nom. Thk. lin.t - cont. Aliow. lin.t

Dism. 1.0. It 4 int

Lenotin (overeall) (1) \& an.)

9. Seams:

$$
-
$$

Sngt, Lap, Burt

$-\frac{-}{\text { EII. }(\text { (x) }}$

$\frac{-}{\text { H.T. Temo. I'FI }}$

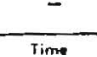

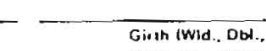
Sngt., Lop, Buri

(b) Matl. $\mathrm{SA} 240-304 \div$

\section{[Spec. No., Gosde)}

\begin{tabular}{|c|c|c|}
\hline $\begin{array}{c}\mid 3 \\
\text { Hemispherical } \\
\text { Ardius }\end{array}$ & $\begin{array}{c}\text { Flar } \\
\text { Oiameles }\end{array}$ & $\begin{array}{c}\text { Side ro Piessure } \\
\text { iConven or Concivel }\end{array}$ \\
\hline- & - & Concave \\
\hline- & - & - \\
\hline
\end{tabular}

\footnotetext{
If removable, bolts used (describe other fastenings)
}

) _-

\section{(Masti, Speec No., Gr., Sire, No.)}

11. Type of Jacket

12. Jacket Closure_

If bolted, describe or sketch.

13. MAWP_ _ psi at max. temp.

Hydro., pneu., or comb. test press.

- _ _ Proot Test

If bar, give dimensions

$-$

\section{ltems 14 and 15 to be completed for tube sections}

14. Tubesheets:
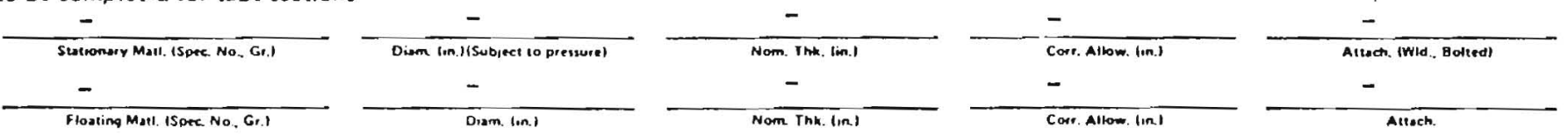

15. Tubes:

Mat. (Sporc. No., Grode)

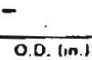

Nom, Thin. (in. or gewpes)
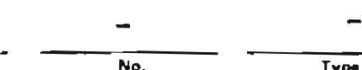

Items 16-18 incl. to be completed for inner chambers of jacketed vesse/s or channels of heat exchangers

16. Shell:

17. Seams:

$$
-
$$

$$
\begin{aligned}
& \text { Lona. Wwid. Dot. } \\
& \text { Sngt. La. Butei }
\end{aligned}
$$

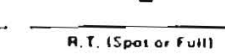

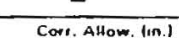

Dism. I.D. IIt sin.1

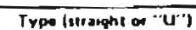

18

18. Heads: (a) Matl.

$\underline{-10}$ $\frac{-}{E^{H+1} \mid(x)}$ $\frac{-}{\text { H. Temp. T.1 }}$
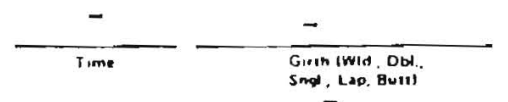

(b) Matl.

-

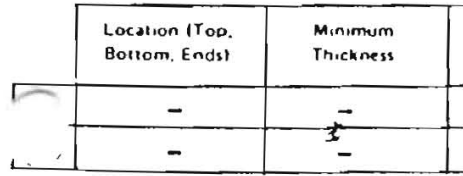

\begin{tabular}{|c|c|c|}
\hline $\begin{array}{c}\text { Corroson } \\
\text { Allowrnce }\end{array}$ & $\begin{array}{c}\text { Crown } \\
\text { Araus }\end{array}$ & $\begin{array}{c}\text { Kouckle } \\
\text { Radus }\end{array}$ \\
\hline- & - & - \\
\hline- & - & - \\
\hline
\end{tabular}

\begin{tabular}{|c|c|c|c|}
$\substack{\text { Elliutical } \\
\text { Ratio }}$ \\
\hline- \\
\hline-
\end{tabular}

$=$

$-1$

16emovable. halts usard (dascritio other fastenings). 
FORM U-2 MANUFACTURER'S PARTIAL DATA REPORT

A Part of a Pressure Vessel Fabricated by One Manufacturer for Another Manufacturer

As Required by the Provisions of the ASME Code Rules, Section VIII, Division 1

1. Manufactured and certified by

The Stacey Mfg. Co., 259 Township Ave., Cincinnati, Ohio 45216

Richmond-Lox Equipment Co., Delphi, Indiana 46923

3. Location of installation___ Not known

4. Trpe Horizontal Tank $\frac{\text { Tank }}{\text { (Horr or vert iank) }}-\frac{6308 B}{\text { (Mlgissetis No. ot Pan) }}-\frac{0-43 A 53 R .2}{\text { (CRN) }}$

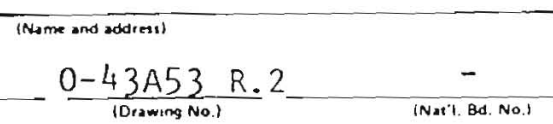

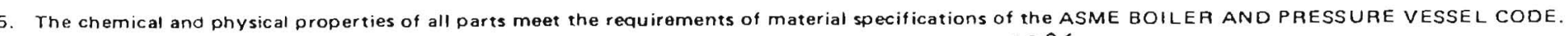
The design, construction, and workmanship conform to ASME Rules, Section VIII, Division 1_ 1986 December 31,1986

Code Case No.

Special service per UG-120(d)

6. (a) Drawing prepared by The Stacey Mfg. Co. (b) Description of part inspected $16: 11-3 / 4^{\prime \prime}$ OD Half Torus*

7. Postweld heat treatment: Temp. ${ }^{\circ} \mathrm{F}$ Time

Items 8-13 incl. to be completed for single wall vessels, jackets of jacketed vessels, or shells of heat exchangers

8. Shell: Mati. ISpec. No., Giadel Nom. Thk. (In.) - Corr. Allow. Iin.t

$$
\text { Mati. ISpec. No., Gradel Nom. Thk. Iin.) Corr. Allow. Iin.) }
$$

9. Seams: Long. (Wid., Dbl. R.Y. (Spol of Full) $-$ H.T. Temp. (F) $-\overline{\text { Time }}$

(b) Matl. Sngt. (ap. Burt

10. Heads: (a) Marl. SA240-304\%

ISpec. No., Grade) (b) Matt. _

\begin{tabular}{|c|c|c|c|c|c|c|}
\hline \multicolumn{5}{|l|}{ No., Grade) } & \multicolumn{2}{|c|}{ (Spec. No., Grade) } \\
\hline $\begin{array}{l}\text { Crown } \\
\text { Padius }\end{array}$ & $\begin{array}{l}\text { Knuckle } \\
\text { Radius }\end{array}$ & $\begin{array}{c}\text { Elliptical } \\
\text { Patio }\end{array}$ & $\begin{array}{c}\text { Conical } \\
\text { Apex Angle }\end{array}$ & $\begin{array}{l}\text { Hemispherical } \\
\text { Radius }\end{array}$ & $\begin{array}{c}\text { Flat } \\
\text { Diameter }\end{array}$ & $\begin{array}{l}\text { Side to Pressure } \\
\text { (Convex or Concave) }\end{array}$ \\
\hline $7^{\prime 3}-7 / 8^{\prime \prime}$ & $1^{\prime} 1-9 / 16^{\prime}$ & $8,5-9 / 16^{\prime \prime}$ & & & & Concave \\
\hline
\end{tabular}

Side to Pressure

If removable, bolts used (describe other fastenings)

(Mall. Spec. No., Gr., Sire, No.)

11. Type of Jacket - Jacket Closure
if bolted, describe or sketch.

13. MAWP

Hydro., pneu., or comb. test press.

Proof Test _ _ -

If bar, give dimensions

${ }^{\circ} \mathrm{F}$. Min. temp. (when less than $-20^{\circ} \mathrm{F}$ ) ${ }^{\circ} \mathrm{F}$.

Items 14 and 15 to be completed for tube sections

14. Tubesheets:
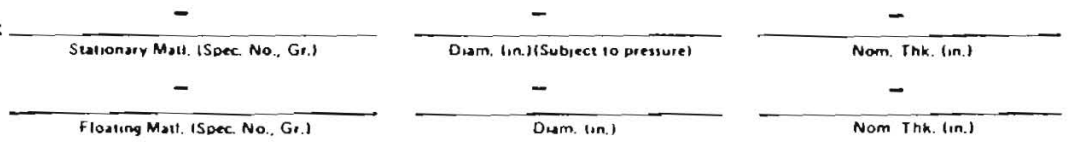
psi.
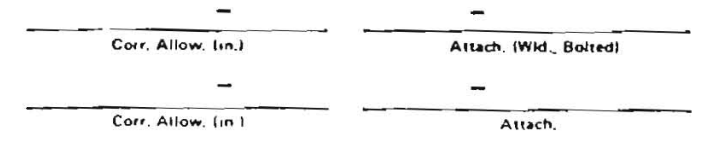

- lin. or gevol

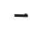

15. Tubes:

$$
\text { Mat. (Spec. No., Grade) }
$$
$\frac{-}{\text { OD. [in.) }}$

ltems 16-18 incl. to be completed for inner chambers of jacketed vessels or channels of heat exchangers

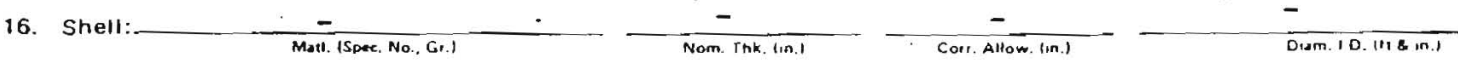

17. Seams:

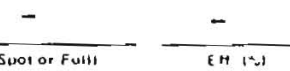
HT. Temp. $1+1$ Time

(b) Matl. ___

18. Heads: (a) Mat1.

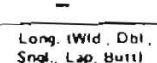

.
$-$

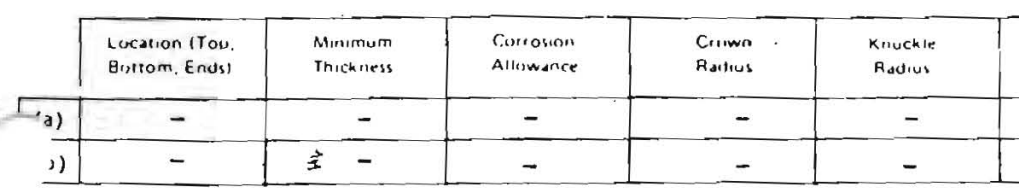

If removable, bolts used (describe other fastenings)

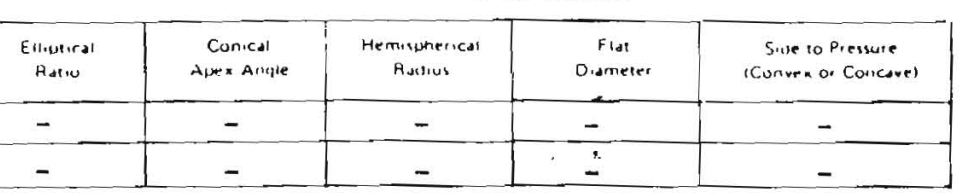

VIat Spec. No Grade Sire No.I 


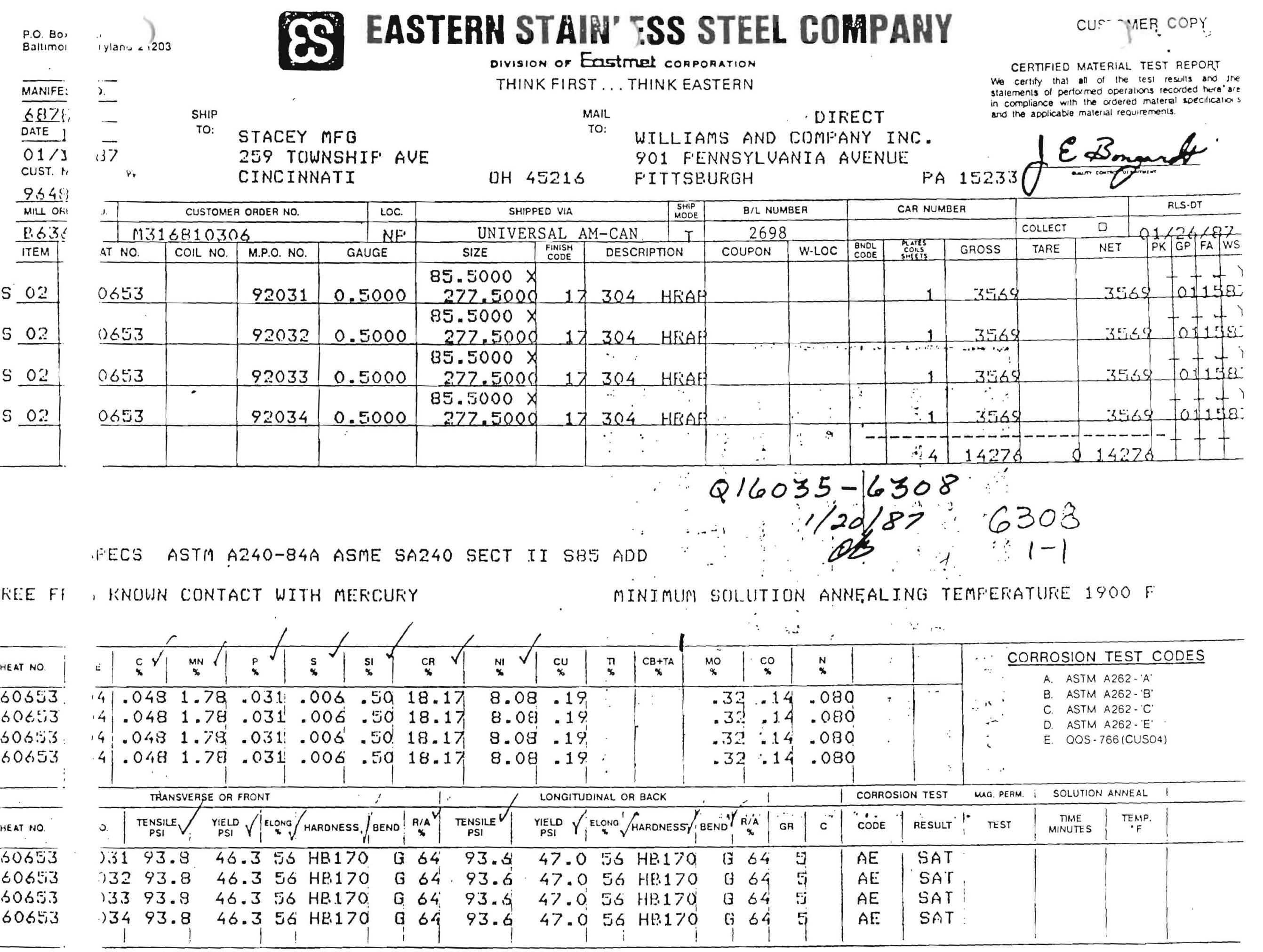

